\title{
Consensus Guidelines for Pediatric Intensive Care Units in India, 2020
}

\author{
Praveen Khilnani, ${ }^{1}$ Bala Ramachandran, ${ }^{2}$ Farhan Shaikh, ${ }^{3}$ Rachna Sharma, ${ }^{4}$ Anil SaChdev, ${ }^{5}$ S Deopujari, ${ }^{6}$ \\ Arun Bansal, ${ }^{7}$ Dayanand Nakate ${ }^{8}$ and Sanjay Ghorpade ${ }^{9}$ for Indian ACademy of Pediatrics College \\ Council of Pediatric Intensive Care Chapter

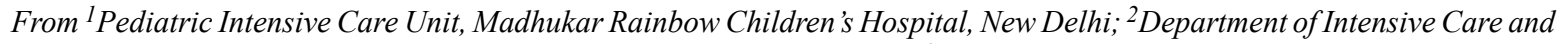 \\ Emergency Medicine, KK CHILDS Trust Hospital, Chennai, Tamil Nadu; ${ }^{3}$ Pediatric Intensive Care Unit, Rainbow Children's \\ Hospital, Banjara Hills, Hyderabad, Telangana; ${ }^{4}$ Pediatric Intensive Care Unit, BLK Superspecialty Hospital, Delhi; ${ }^{5}$ Pediatric \\ Intensive Care Unit, SGRH, Delhi; ${ }^{6}$ Nelson Child Hospital, Nagpur Maharashtra; ${ }^{7}$ Department of Pediatrics and Pediatric Critical \\ Care, PGIMER, Chandigarh; ${ }^{8}$ Ashwini Sahakari Rugnalaya Ani Sanshodhan Kendra Nyt, Solapur, Maharashtra; and \\ ${ }^{9}$ Pediatrician, Satara, Maharashtra; India. \\ Correspondence to: Dr Praveen Khilnani, Director, Pediatric Critical Care and Emergency Services, Madhukar Rainbow \\ Children's Hospital, Malviya Nagar, NewDelhi110 017,India.praveenkhilnanil957@gmail.com
}

\begin{abstract}
Background: Consensus Guidelines for Pediatric Intensive Care Units (PICUs) were published in Indian Pediatrics in 2002. Objective: The current document represents a recent update in the Indian context, regarding unit design, equipment, organization, staffing as well as admission and discharge criteria for different levels of Pediatric Intensive Care and teaching units with PICU training programs, as well as nonteaching units. Process: The Pediatric Intensive Care College Council (PICC), an academic wing of the Indian Academy of Pediatrics (IAP) Intensive Care Chapter took the initiative to update the guidelines with members of the PICU guidelines Committee writing group. After a great deal of discussion at conferences and through mailing and feedback with listed members, as well as with the guidance and feedback of senior PICU guidelines advisory committee members, The consensus is now updated. These guidelines are intended to serve as a reference for health Care institutions wishing to establish a new PICU or to modify an existing PICU. As a resource, experience of those members who have worked extensively in western PICUs was also taken into consideration, in addition to published guidelines in the medical literature. PICUs with teaching programs run by the IAP Intensive Care Chapter must follow these criteria for unit accreditation and teaching curricula as applicable. Recommendations: Unit design, equipment, organization, staffing as well as admission and discharge criteria for different levels of pediatric intensive care are updated.
\end{abstract}

Keywords: Accreditation, Criteria, Critical care, Design, Level of care.

Published online: August 29, 2020. PII: S097475591600237

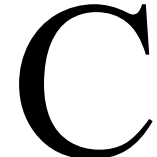
onsensus Guidelines for pediatric intensive care units (PICUs) were published in Indian Pediatrics in 2002 [1]. The current document represents a recent update in the Indian context, regarding unit design, equipment, organization, staffing as well as admission and discharge criteria for different levels of pediatric intensive care. The Pediatric Intensive Care College Council (PICC), the academic wing of the Indian Academy of Pediatrics (IAP) Intensive Care Chapter, undertook the task of updating the guidelines. These guidelines can serve as a reference for health care institutions wishing to establish a new PICU or to modify an existing PICU.

\section{PROCESS}

The PICC, an academic wing of IAP Intensive Care Chapter took the initiative to update the earlier PICU guidelines 2002 [1] by formation of PICU guidelines committee with leadership and members of accreditation committee of PICC, IAP Intensive Care Chapter on 30
June, 2019 at Rainbow Children's Hospital, Hyderabad. A writing group (PICU guidelines advisory committee was also constituted. Advisory committee constituted senior members who have been closely involved with the development of Pediatric Intensive Care Units at both Governmental and Non-Governmental hospitals in India since the inception of the IAP Intensive Care Chapter. During this discussion due consideration was given to adequately develop and adapt the guidelines to be applicable in the Indian context [2]. As a resource, experience of those members who have worked extensively in Western PICUs was also taken into consideration, in addition to incorporating information from published guidelines in the medical literature [2-8]. After a great deal of discussion and through mailings and feedback with listed members, the consensus was achieved on 24th July, 2019. The consensus achieved was then taken up by guidelines writing group, which prepared these guidelines. 


\section{RECOMMENDATIONS}

\section{Unit Design}

The PICU should be dedicated for infants and children, separate from the neonatal and adult ICU [3]. The Unit should be preferably located near the lift, with easy access to the emergency department, operation theatre, laboratory and radiology departments.

The doctors' duty room as well as consultant intensivist's office and counselling room should be close to the PICU, with intercom facility. Other facilities nearby should include a staff area with locker cabinets, a family waiting area to provide for at least one (preferably two) person per admitted patient with bathroom, shower and telephone facility, as feasible.

\section{Size of PICU}

Six to twelve beds is desirable. PICUs with less than 4 beds risk inefficiency and PICUs with greater than 16 beds may be difficult to manage, if not properly divided [3]. For the total Pediatric ward beds up to 25, a PICU of six to eight beds is ideal (4:1). Additional beds and separate units may be required if specialized surgery such as heart surgery, multi organ transplant surgery, neurosurgery and trauma surgery cases are routinely expected. In addition an oncology and bone marrow transplant unit may also be required at tertiary care centers to account for total numbers of ICU beds.

\section{Room Layout and Bed Area}

Ideally layout should allow actual visualization of all patients from central station; however, a central monitoring station is essential even if direct visualization of the patient from central station is not feasible in order to have a wholesome and close monitoring

Patient area in open PICU should be $100-150$ sqft. In a cubicle, the minimum area should be 125 to $200 \mathrm{sqft}$. with at least one wash basin facility for two beds. Ideally, one for each bed is preferred. At least one, preferably two rooms should have an isolation capability with an area of 250 square feet with an ante room (separate area at least 20 square feet for hand washing and wearing mask and gown) and to provide true airborne isolation capability with negative pressure ventilation.

The area around the bed should allow enough space for performing routine ICU procedures such as central lines, chest tube placement, as well as for easy access for portable $X$-ray machine, portable ultrasound, electrocardiograph and portable electroencephalograph machine. An easy access to head end of the patient for emergency airway management is a must on all beds. Removable head board should be available in PICU beds for easy access to airway intervention. Wall and ceilings should be constructed of materials with high sound absorption capabilities. Walls, ceilings and the floor should be smooth, non-porous and easy to clean. All edges must be coved to minimize accumulation of dust. Wall oxygen outlets (two), air outlet (two), two suction outlets, and at least ten electrical outlets per bed are recommended for various equipment $[3,4]$. In rooms, windows are preferable to prevent a sense of isolation. Adequate lighting, child friendly wall papering or paintings with soothing colors on walls/ceiling and soothing color on curtains are desirable.

\section{Power Supply and Temperature Control}

Unit should preferably be centrally air conditioned and should have central heating for temperature control. Air conditioning should be designed so that air flow is always from a clean to dirty area. In case of lack of central heating system, overhead warmers should be available. Unit should have an uninterrupted power supply by means of backup power sources such as invertors and generators in accordance with load requirement of various equipment.

\section{Beds}

Beds should have ability to manoeuvre head end and foot end as well as availability of two or more air/water mattresses to prevent bed sores. All beds must have a railing to prevent accidental fall of the child. Each bed should have an emergency alarm button for the nurse or intensivist to activate code system [4] for emergencies. An intercom at each bed is desirable. A cart with closet drawer at the bedside is important to hold personal belongings and required patient items.

\section{Crash Cart and Work Area}

A crash cart with all standard emergency drugs and portable monitor/defibrillator should be readily accessible. Zones should be provided for medication preparation and cabinets should be available for the storage of medications and supplies.

A PICU receptionist area is ideal to control visitation so that all visitors must go via this area before entering then ICU. This area should be monitored by security personnel.

\section{Central Station [5]}

A central station should provide visibility to all patient areas [5]. It should have ample area to have capacity for all necessary staff functions. Patient records should be easily available. Adequate space for computers, printers and central monitor is essential. Ample space for staff to write on patient files, and space for unit secretarial staff is 
Table I PICU Levels of Care: Design, Equipment, and Support Services

\begin{tabular}{|c|c|c|}
\hline Design & Level 3 & Level 2 \\
\hline Rooms & $\begin{array}{l}\text { Conference/duty room mandatory. Clean and dirty utility rooms } \\
\text { mandatory. Library desirable.Toilet for patients mandatory }\end{array}$ & $\begin{array}{l}\text { Conference/duty room mandatory. Toilet } \\
\text { for patients mandatory }\end{array}$ \\
\hline Spacing & $\begin{array}{l}\text { Ward type beds: } \min .100 \text { sq.ft/ bed ( } 150 \text { sq. } \mathrm{ft} \text { desirable; } \\
\text { cubicle: } \min .125 \mathrm{sq} . \mathrm{ft} / \text { bed }(200 \text { sq. } \mathrm{ft} \text { desirable })\end{array}$ & Not specified \\
\hline \multicolumn{3}{|c|}{ Equipment and monitoring } \\
\hline Monitoring & $\begin{array}{l}\text { ECG, } \mathrm{RR}, \mathrm{SpO}_{2}, \mathrm{NIBP} \text { for all beds. Invasive BP monitoring : } \\
\text { at least } 50 \% \text { of beds }\end{array}$ & $\begin{array}{l}\text { ECG, RR, } \mathrm{SpO}_{2}, \mathrm{NIBP} \text { for at least } 50 \% \\
\text { beds; } \mathrm{SpO}_{2} \text { for all other beds }\end{array}$ \\
\hline Ventilator & $\begin{array}{l}\text { Compulsory: Invasive ventilators, NIV and high flow nasal } \\
\text { cannula (HFNC); Desirable: High frequency oscillatory } \\
\text { ventilation (HFOV) }\end{array}$ & $\begin{array}{l}\text { Compulsory: Invasive ventilator; } \\
\text { Desirable: N on Invasive Ventilation } \\
\text { (NIV)/HFNC }\end{array}$ \\
\hline Equipment & $\begin{array}{l}\text { Mandatory: Infusion pumps, Warmers, Neonatal open care } \\
\text { systems, EEG facility, Defibrillator }\end{array}$ & $\begin{array}{l}\text { Mandatory: Infusion pumps, Warmers, } \\
\text { Defibrillator }\end{array}$ \\
\hline Crash cart & Appropriately stocked crash cart mandatory & Appropriately stocked crash cart mandatory \\
\hline \multicolumn{3}{|l|}{ Ancillary services } \\
\hline Lab facility & $\begin{array}{l}\text { In house and } 24 \text { hour for CBC, RFT, LFT, Coagulation studies, } \\
\text { ABG and lactate. Other Investigations can be outsourced }\end{array}$ & In house and 24 hour for $\mathrm{CBC}, \mathrm{RFT}, \mathrm{ABG}$. \\
\hline Support services & $\begin{array}{l}24 \text { hours access to blood bank, Pharmacy, Neurosurgery, } \\
\text { Pediatric surgery and ENT surgical facilities }\end{array}$ & $\begin{array}{l}24 \text { hours access to blood bank, pharmacy } \\
\text { and Pediatric surgery }\end{array}$ \\
\hline $\begin{array}{l}\text { Quality improve- } \\
\text { ment (QI) }\end{array}$ & $\begin{array}{l}\text { Regular audit of key QI data including Catheter associated } \\
\text { urinary tract infection (CAUTI), Central line associated } \\
\text { blood stream infection (CLABSI), Ventilator associated } \\
\text { pneumonia (VAP) rates, medication errors, readmission, and } \\
\text { re-intubation rates; Must use a severity of illness scoring } \\
\text { (PRISM or PIM) }\end{array}$ & $\begin{array}{l}\text { Desirable: Regular audit of key QI data } \\
\text { including CAUTI, CLABSI, VAP rate, } \\
\text { medication error, readmission, and } \\
\text { re-intubation }\end{array}$ \\
\hline
\end{tabular}

essential. At least two telephone lines should be available. A cordless telephone instrument is desirable for the nurse in-charge and for the PICU doctor on duty. If possible, a telephone line may be dedicated to incoming calls only to facilitate critical care transport requests or other urgent calls.

\section{Imaging Film Viewing Area}

A distinct area in PICU should be chosen for viewing and storage of imaging films. An illuminated viewing box should allow viewing of several images as well as for comparison.

\section{Storage}

Storage for vital supplies should be located within or closely adjoining to PICU. A refrigerator is essential for some pharmaceutical products. An area must be provided for storage of large patient care equipment items not in active use. An area must be provided for stretchers and wheel chairs.

\section{Clean and Dirty Utility Room}

Clean and dirty utility rooms must be separate. The clean utility room should be used for the storage of clean linen.
Dirty utility room must contain a separate sink. Covered bins must be provided for soiled linen and waste materials. An area for emptying and cleaning bed pans and urine bottles is also necessary. The dirty utility area and toilets should have independent exhausts that cannot be switched off. Exhaust function should have visible indicators (flutter strips).

\section{Waste Disposal}

Mechanism of disposal of contaminated waste (segregation of garbage and contaminated medical waste) and adequate disposal of needles and sharp objects needs to be as per standard applicable pollution control guidelines [6].

\section{Conference Room and Library}

A room for intensivist and staff for education, discussion of difficult cases and other necessary meetings related to quality improvement is desirable. This room should have a small library facility with ready access to topical scientific literature. It should also have a computer with reliable internet access to facilitate access to various online resources, and/or to point of care management tool. 


\section{Counselling Room}

A room for intensivist and parents for regular counselling sessions regarding progress of patient condition and plan of treatment is important. Audio-video recording facility to record the counselling is highly recommended, with prior disclosure and discussion with family. Counselling session clips should be preserved for minimum of 5 years.

\section{Urgent Laboratory}

A laboratory (stat Laboratory) with quick turnaround time (less than one hour) for urgent investigations such as arterial blood gas, electrolyte, blood sugar, urea, creatinine, prothrombin time, partial thromboplastin time, complete blood count and urine examination with Gram stain should be available. Point of care portable equipment such as i-Stat is also acceptable (if available). Twenty four hour availability of on site or in hospital arterial blood gas is essential.

\section{Equipment}

The selection of equipment should be based on: cost benefit analysis; accuracy and adaptability for pediatric population; ease of use for care givers; troubleshooting requirements; proven use on pediatric patients; maintenance requirements; availability of biomedical support in the hospital. It is important to obtain user list before buying new equipment first for after sale service and to identify problem with equipment if any. The list of recommended equipment for a tertiary level PICU is provided at the society's website (www.PICCIndia.org). Emergency (crash) cart should be regularly checked with documentation of date, time and person who checked and setting up of a process immediate and ongoing replacement of used item or drugs on a regular basis.

\section{ORGANIZATION AND STAFFING}

\section{Medical Director/Intensivist Incharge [5]}

The medical director/intensivist incharge should be a Pediatrician fully qualified and trained with experience in delivery of comprehensive critical care of children with the following responsibilities:

- Establishing policies and protocols with the help of a group of experts including but not limited to pediatric consultants and subspecialists, nursing director, administration, laboratory and blood bank representatives as per prevalent norms, as well as using information from existing published guidelines; for example guidelines from the CDC (Centers for Disease Control) for infection control or international surviving sepsis guidelines.

- Smooth functioning of PICU with implementation of policies and protocols including admission and discharge criteria.

- Quality assurance and continuous quality improvement (CQI) (committee membership).

- Advice hospital administration regarding equipment needs.

- Establishing teaching and training system of medical, nursing and ancillary staff.

- Maintaining PICU statistics for mortality and morbidity.

- Active membership of hospital infection control committee (HICC).

- To conduct regular quality improvement meeting including mortality and morbidity meetings to especially analyse infection control and outcome data.

\section{Staffing Requirements}

Medical staff: The medical staff should consist of round the clock coverage by post graduate level pediatrician in the PICU with good airway and pediatric basic (BLS) and advanced life support (ALS) skills and active currently valid ALS certification (PALS/IAP-ALS).

Nursing staff: A qualified experienced nursing manager is essential. Adequate nursing staff with all shifts fully covered, is an essential requirement for good quality patient care. All ventilated patients need one Pediatric ICU trained nurse by the bed side (1:1). A very unstable patient (hypotensive/hypoxemic patient despite moderate support) may require two nurses by the bed side $(2: 1)$ or more. Other unventilated/relatively stable patients (such as post-operative patients and ones admitted for overnight observation) may require only one nurse per 23 patients (1:2-3).

\section{Ancillary support services}

\section{Ancillary Staff}

All PICUs must be regularly staffed by physiotherapists, dieticians and respiratory technicians for enhancing patient care. In addition, technicians, radiographers, and biomedical engineers should be available on a 24 hours (in hospital) basis for emergencies/problems that require immediate attention such as power failure, central gas supply problems, malfunctioning equipment, or need for urgent $X$-ray of chest in a patient with suspected pneumothorax or CT-scan of head spine, thorax or abdomen, as the case may be. Secretarial/clerical staff is essential to carry out communication as well as paper work necessary for smooth functioning of the Unit. It is 
also essential to have cleaning staff that is efficient and sensitive to urgent patient care needs, in addition to regular cleaning and mopping the floor. Presence of social service personnel is desirable to help support families emotionally as well as financially in stressful circumstances.

\section{Levels of PICU Care}

Two levels of PICU care are identified, level 3 and level 2. Level 3 (tertiary) PICU can be organized with a level 2 (step down/high dependency) service in nearby but separate area. In small private setups, level 3 and level 2 care can be provided in one unit if facilities and equipment as well as personnel as described below are available. These criteria for level 2 units are given in $\mathbf{W e b}$

Table I.

\section{Level 3 Care (Tertiary level PICU) Requirements}

(a) Defined admission, discharge policies;

(b) Four to six ventilator beds;

(c) More than 200 admissions per annum;

(d) Pediatric intensivist heading the unit;

(e) One pediatrician with post graduate training and experience in critical care present in PICU at all times;

( $f$ ) Minimum one on one nursing on ventilated patients;

(g) High level of monitoring capability in all patients;

(h) 24 hour access to blood bank, pharmacy, pathology, operating theatre, and tertiary level imaging services;

(i) Educational and research activities; and

(j) Quality review/audit process in place.

\section{Quaternary Facility/Specialized PICU Level of Care [7]}

A quaternary PICU facility is defined as one that is commonly found in university or children's hospitals that provide regional care and serve large populations or have a large catchment area in Western countries. The center would provide comprehensive care to all complex patients, including but not limited to those with significant cardiovascular disease, end-stage pulmonary disease, complex neurologic/neurosurgical issues, transplantation services (both bone marrow transplant and solid organ), ECMO (extra corporeal membrane oxygenation), multisystem trauma, and burns greater than $10 \%$ total body surface area. A specialized PICU provides diagnosis-specific care for select patient populations. Examples of this might include a cardiac ICU or a burn unit that provide pediatric critical care.
Box I Admission Criteria to level 3 Care PICU

All patients requiring mechanical ventilation

Patients with impending respiratory failure

- Upper airway obstruction

- Lower airway obstruction

- Alveolar disease

- Unstable airway

All pediatric patients after successful resuscitation

Comatose patients

- Meningitis, encephalitis

- Hepatic encephalopathy

- Cerebral malaria

- Head injury

- Poisonings

- Status epilepticus

All types of shock/hemodynamic instability

- Septic shock

- Hypovolemic shock

- Bleeding emergencies such as gastrointestinal (GI) bleeding, bleeding diathesis, Disseminated Intravascular Coagulation (DIC)

- Cardiogenic shock - myocarditis, cardiomyopathy, congenital heart disease

- Neurogenic shock

- Multiple trauma

Cardiac arrhythmias

- Hypertensive emergencies

- Severe acid base disorders

- Severe electrolyte abnormalities

Acute renal failure

- Patients requiring acute hemodialysis

- Hemofiltration-peritoneal dialysis

Post-operative patients

- Requiring ventilation

- Unstable patients

- Post-operative patients after open heart surgery, neurosurgery, thoracic surgery and other patients after major general surgery with potential for respiratory/ hemodynamic instability (may go to dedicated unit if available)

Patients requiring ECMO (Extra corporeal membrane oxygenation), Nitric Oxide therapy

Malignant hyperpyrexia

Acute hepatic failure

All post-transplant patients (if applicable)

These ICUs have specialized equipment and supplies as well as medical, nursing, and other members of the patient care team with specific skills dedicated to a certain discipline. Such units are few in number but slowly coming up in various parts of our country. Currently our guidelines do not distinguish quaternary level from tertiary care level 3 units.

\section{Admission and Discharge Criteria [8]}

The suggested admission criteria to level 3 care PICU are 


\section{Box II Admission Criteria to Level 2 Care (Step Down Unit /High Dependency Unit)}

All ward patients requiring close monitoring due to potentially unstable conditions;

Croup (laryngotracheobronchitis) requiring oxygen;

Asthma requiring hourly nebulization/getting tired with increasing oxygen requirement/mental status change;

All patients requiring more than $50 \%$ oxygen to maintain saturations;

Closed head injury/skull fracture admitted for observation;

Diabetes ketoacidosis with $\mathrm{pH}<7.2$;

Patients with episodes of apnea;

Patients with significant abdominal trauma with suspected renal/splenic/hepatic injury;

Severe dehydration with mental status change;

Post-operative patients after major surgery with significant post-operative pain/blood loss/stress;

Patients recovering from critical illness (level 3 Care), but requiring close monitoring

shown in Box I and in Box II for Level 2 care (Step down unit/High-dependency unit).

\section{List of Recommended Drugs to be Available}

Web Annexure I shows a list of drugs that should be available in the PICU. The list may vary depending on the availability; however, essential emergency drugs must be available round the clock. Crash cart medications should be replaced immediately and crash cart should be maintained on a regular basis with respect to equipment, oxygen cylinder, laryngoscope, lights, battery, defibrillator and other essential material.

\section{CONCLUSIONS}

All recommendations concerning pediatric intensive care units in India, including unit design, equipment, organization, staffing as well as admission and discharge criteria for different levels of pediatric intensive care are revised and updated as of year 2020 .

Disclaimer: These recommendations are to be considered as guidelines in the strict sense and by no means an established standard of care for all PICUs in India.

Competing interests: None stated; Funding: None.

\section{REFERENCES}

1. Khilnani P. Consensus Guidelines for Pediatric Intensive Care Units in India. Indian Pediatr. 2002;39:43-50.

2. Khilnani P. Pediatric critical care in the 21 st century. Indian J Pediatr. 1998;65:707-08.

3. Driscoll S, Flemming M, Khilnani P. Establishing a new pediatric intensive care unit. Indian J Pediatr. 1993;331-39.

4. Yeh TS. Issues in unit management and design. In: Fuhrman B, Zimmerman J, eds. Pediatric Critical Care. 1995, Mosby;51-8.

5. Task Force on Guidelines, Society of Critical Care
Medicine. Recommendations for critical care unit design. Crit Care Med. 1988;16:796-808.

6. Central Pollution Control Board, Ministry of Environment, Forest \& Climate Change and Directorate General of Health Services, Ministry of Health \& Family Welfare. Guidelines for the Management of Healthcare Waste as per Biomedical Waste Management Rules, 2016. Chapter 2:2.2:6.

7. Rosenberg DI, Moss MM. American Academy of Pediatrics Section on Critical Care; American Academy of Pediatrics Committee on Hospital Care. Guidelines and Levels of Care for Pediatric Intensive Care Units. Pediatrics. 2004;114:1114-25.

8. Frankel LR, Hsu BS, Yeh TS, Simone S, D'Agus NSm Area MJ, et al. Criteria for Critical Care Infants and Children: PICU Admission, Discharge, and Triage Practice Statement and Levels of Care Guidance. Pediatr Crit Care Med. 2019;20:847-87.

\section{Annexure I}

\section{PICU Guidelines Advisory Committee Members}

Shekhar Venkataraman, Pennsylvania, USA (Mentor: IAP College Council of Pediatric Intensive Care Chapter); Bakul Parekh, Mumbai, Maharashtra (President Central IAP 2020 and Ex-officio Chair IAP, Intensive Care Chapter and Chancellor IAP College Council of Pediatric Intensive Care Chapter, 2019); GV Basavaraja, Bangalore, Karnataka (Chair Elect 2020 IAP Intensive Care Chapter. Secretary General IAP 2020 IAP College Council of Pediatric Intensive Care Chapter); S Soans, Mangalore, Karnataka (Central IAP President 2018); S Singhi, Gurugram, Haryana (Advisor IAP College Council of Pediatric Intensive Care Chapter); K Chugh, Gurugram, Haryana (Advisor IAP College Council of Pediatric Intensive Care Chapter); Soonu Udani, Mumbai, Maharashtra (Advisor IAP College Council of Pediatric Intensive Care Chapter Director); Suchitra Ranjit, Chennai, Tamil Nadu (Advisor IAP College Council of Pediatric Intensive Care Chapter); Rajiv Uttam, Delhi (Joint Secretary IAP College Council of Pediatric Intensive Care Chapter). 